\title{
Deletion of the fibrogen alpha-chain gene (FGA) causes congenital afibrogenemia
}

\author{
Marguerite Neerman-Arbez, Ariane Honsberger, Stylianos E. Antonarakis, \\ and Michael A. Morris \\ Division of Medical Genetics, University Medical School and University Hospital, CH-1211 Geneva, Switzerland \\ Address correspondence to: Marguerite Neerman-Arbez or Michael Morris, Centre Médical Universitaire, 1 rue Michel-Servet, \\ CH-1211 Geneva, Switzerland. Phone: 41-22-702 56 92; Fax: 41-22-702 57 06; E-mail: Marguerite.Arbez@medecine.unige.ch \\ or Michael.Morris@medecine.unige.ch
}

Received for publication October 9, 1998, and accepted in revised form December 1, 1998.

\begin{abstract}
Congenital afibrinogenemia is a rare autosomal recessive disorder characterized by the complete absence of detectable fibrinogen. Uncontrolled bleeding after birth from the umbilical cord is common, and spontaneous intracerebral bleeding and splenic rupture can occur throughout life. Patients respond well to fibrinogen replacement therapy, either prophylactically or on demand. Because the half-life of infused fibrinogen is essentially normal, the genetic defect is assumed to be at the level of synthesis, but no responsible locus has been identified. Preliminary studies using Southern blotting suggested that no gross structural changes of the fibrinogen genes were present in patients. We report the identification of causative mutations in a nonconsanguineous Swiss family with congenital afibrinogenemia. The four affected male individuals (two brothers and their two first cousins) have homozygous deletions of $\sim 11 \mathrm{~kb}$ of the fibrinogen alpha-chain gene (FGA) Haplotype data suggest that these deletions occurred separately, on three distinct ancestral chromosomes, implying that the FGA region of the fibrinogen locus is susceptible to deletion by a common mechanism. Furthermore, our results demonstrate that humans, like mice, may be born without the capacity to synthesize functional fibrinogen.

J. Clin. Invest. 103:215-218 (1999).
\end{abstract}

\section{Introduction}

The two alternative pathways of blood coagulation, the intrinsic and the extrinsic, converge with the proteolytic conversion of prothrombin to thrombin, the serine protease that is, in turn, responsible for activating fibrinogen and producing a fibrin clot. Genetic abnormalities have been described at many levels of these pathways: for example, the absence of key cofactors (hemophilia A; ref. 1), the production of abnormal proteins (factor V Leiden; ref. 2), or disorders of protein secretion (combined factor $\mathrm{V}$-factor VIII deficiency; ref. 3). Congenital afibrinogenemia (Mendelian Inheritance in Man No. 202400) was originally described in 1920 (4), and to date some 150 families with this disorder have been described in the literature (5). As is commonly the case with rare autosomal recessive disorders, $\sim 50 \%$ of cases are associated with consanguinity (6). Although functional assays of clot formation are infinitely prolonged in affected individuals, the coagulation defect is surprisingly no more severe than in the hemophilias A and B, varying from severe to mild. Uncontrolled bleeding after birth from the umbilical cord is common, and spontaneous intracerebral bleeding and splenic rupture can occur throughout life. Patients respond well to fibrinogen-replacement therapy. The genetic defect is assumed to be at the level of fibrinogen synthesis, because the half-life of infused fibrinogen is essentially normal, but no responsible gene has been identified to date.

The fibrinogen locus comprises three genes coding for fibrinogen gamma $(F G G)$, alpha $(F G A)$, and beta $(F G B)$, clustered in a region of $\sim 50 \mathrm{~kb}$ on chromosome 4q28-q31 (7). Early studies using Southern blotting sug- gested that no gross structural changes of the fibrinogen genes were present in patients (8). In contrast, in various forms of dysfibrinogenemia, many missense mutations have been found in the syntenic genes for the gamma, alpha, and beta subunits of fibrinogen ( 300 abnormal fibrinogens have been reported, with some 83 structural defects identified; ref. 5). We studied this region in a Swiss family with two pairs of affected brothers using microsatellite analysis, PCR amplification, and Southern blotting. We found that the genetic defect in this family was a recurrent deletion of $\sim 11 \mathrm{~kb}$ of DNA that eliminates the majority of the FGA gene and so leads to an absence of functional fibrinogen.

\section{Methods}

Chromosome 4 microsatellite analysis. DNA extractions and microsatellite analysis were performed as previously described (9). The polymorphic loci used for haplotype analysis around the fibrinogen gene cluster on chromosome 4 were: D4S1625, D4S2962, FGA-i3 (a TCTT repeat located in intron 3 of the fibrinogen alpha gene), D4S2934, and D4S1629 (ordered from centromere to telomere, according to the Marshfield map, http://www.marshmed.org/genetics/).

PCR amplification of selected fibrinogen exons. PCR was performed from genomic DNA under standard conditions. Primers were designed from the sequence for each fibrinogen gene obtained from Genbank (accession Nos.: alpha: M64982; beta: M64983; gamma: M10014). These primers are listed in Table 1.

Southern blotting. Genomic DNA $(1-2 \mu \mathrm{g})$ was digested with BamHI, and Southern blotting was performed according to standard techniques. The PAFS1 probe was produced by PCR amplification of a 516-bp sequence from the intergenic region 


\section{Figure 1}

(a) Pedigree of the Swiss congenital afibrinogenemia family and haplotypes of the region surrounding the fibrinogen locus. The markers (from top to bottom) and genetic distances were: D4S1625 - (13\%) - D4S2962 - (2\%) - FGAi3 - (2\%) - D4S2934 - (2\%) - D4S1629. The FGA deletion (shown as $\Delta$ ) is associated with three different haplotypes. (b) PAGE analysis of the tetranucleotide repeat marker FGAi3, which is homozygously deleted in individuals 6-9. FGA, fibrinogen alpha-chain gene.

between the FGG and FGA genes (Genbank accession No. U36478). The primers used were $\left(5^{\prime} \rightarrow 3^{\prime}\right)$ F: GGAAGTCTGTTCTGGTGTGC, and R: CCTATAATGTGCGTGATCTAG. The PCR product was then cloned by TOPO-TA cloning (Invitrogen Corp., Carlsbad, California, USA).

\section{Results}

The fibrinogen locus is comprised of single copies of three genes coding for fibrinogen gamma $(F G G)$, alpha $(F G A)$, and beta $(F G B)$, clustered in a region of $\sim 50 \mathrm{~kb}$ on chromosome 4q28-q31 (7). We studied this region in a Swiss family with two pairs of affected brothers (Fig. 1a) using microsatellite analysis, PCR amplification, and Southern blotting. Despite intensive genealogical investigation, no consanguinity was identified in the family. The diagnosis of afibrinogenemia for all the affected individuals was made in the neonatal period, after bleeding from the umbilical cord. All four patients (now 30-38 years old) were first treated with purified fibrinogen only after trauma or before operative surgery, a strategy replaced in early adulthood by a prophylactic protocol consisting of a fibrinogen infusion every two to four weeks. Anti-fibrinogen antibodies have never been detected in any of the patients. All coagulation tests performed (activated partial thromboplastin time, thrombin time, prothrombin time, fibrinogen Clauss, and reptilase) were compatible with the complete absence of active fibrinogen, the blood being uncoagulable. Furthermore, no fibrinogen was detectable by Laurell electroimmunoassay in plasma from affected individuals.

Figure 1 shows the family tree, together with haplotype data obtained for five microsatellite markers (D4S1625, D4S2962, FGAi3, D4S2934, D4S1629) covering a region of $15-20 \mathrm{cM}$ around the fibrinogen gene cluster. One of these, FGAi3, a (TCTT)n polymorphic marker situated in intron 3 of the FGA gene, was homozygously deleted in all four affected individuals and was

Table 1

Sequences of the PCR primers used to evaluate the extent of the deletion

$\begin{array}{llr}\text { Target sequence } & \text { Forward }\left(5^{\prime} \rightarrow 3^{\prime}\right) & \text { Reverse }\left(5^{\prime} \rightarrow 3^{\prime}\right) \\ F G G \text { exons 1-2 } & \text { GACACTACAAGGCTCGGAGC } & \text { GATGCAGCAGTTGTCTCTGG } \\ F G G \text { exon } 7 & \text { AGACTTGATGGCAGTGTAG } & \text { CTGGTTCTGCCATTCCAGTC } \\ F G A \text { exon } 1 & \text { CAGCCCCACCCTTAGAAAAG } & \text { CCTGGGTCATAAAGCTAAG } \\ F G A \text { intron 1 } & \text { (as above) } & \text { CTACTGTAGAGCTCTTTCTCC } \\ F G A \text { exon 2 } & \text { CCTCTTCTGGCTAACATTGC } & \text { AGAGGGAGGAATCTCCTGC } \\ F G A \text { exon } 3 & \text { ACAAATGCCCTTCTGGCTGC } & \text { TTGGCTGAGGAAAATCGCC } \\ F G A \text { exon } 5 & \text { GATCTTGTCGAGGGTATGC } & \text { GCGGCATGTCTGTTAATGCC } \\ F G B \text { exon 2 } & \text { TTTCTTCAGTGCCCGTGGTC } & \text { GTCTGGTCAGCGTAAGAC } \\ F G B \text { exon 7 } & \text { TAGCCAGCTTACCAGGATGG } & \text { TGTCTCTGTCATACGTGCTG }\end{array}$

$F G A$, fibrinogen alpha-chain gene; $F G B$, fibrinogen beta-chain gene; $F G G$, fibrinogen gamma-chain gene.
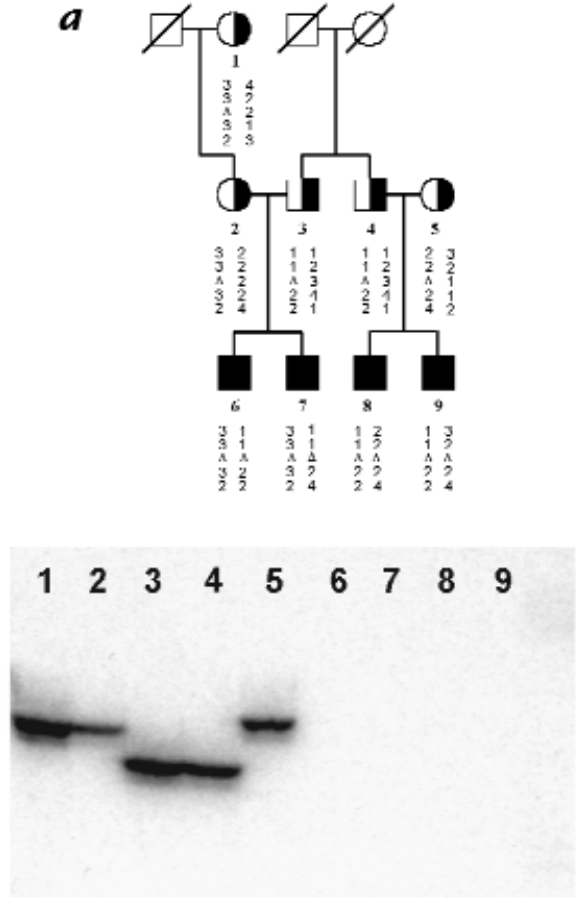

hemizygous in the obligate carriers, providing the first identification of the causative mutation.

The haplotypes of the mutated chromosomes were determined by studying four further polymorphic genetic markers, two to each side of the fibrinogen gene cluster. Three of these four flanking markers were very closely linked to fibrinogen: the genetic distances were D4S1625 - (13\%) - D4S2962 - (2\%) - fibrinogen - (2\%) D4S2934 - (2\%) - D4S1629. The haplotype data reveal that the deletions were present on three different chromosomes, with the haplotypes 3-3-del-3-2, 2-2-del-2-4, and 1-1-del-2-2 (the last being shared by the two fathers of the affected patients; Fig. 1). This implies either that the deletions occurred as separate historical events on three different ancestral chromosomes, or that the mutation occurred originally on a single founder chromosome that produced the three observed haplotypes by repeated recombination. In our opinion, the hypothesis of a common molecular mechanism leading to recurrent mutation is more likely, given the number of recombination events between closely linked markers that would be necessary to generate the observed haplotypes. To characterize the extent of the deletions, we performed PCR amplification of portions of the three fibrinogen genes. Bands of the predicted sizes were obtained for all individuals for $F G G$ exons 1, 2, and 7, FGA exon 1, and FGB exons 2 and 7 (details of the primers are in Methods). PCR amplification of FGA exons 2, 3 , and 5 resulted in bands of the expected size from heterozygous carriers, but in contrast, no amplification was obtained from DNA from all four patients (Fig. 2, and not shown). These results indicate that the centromeric breakpoint of all the deletions is within FGA intron 1. Consequently, the deleted gene could at best direct the production of a severely truncated 
FGA polypeptide, containing just the 18 amino acids encoded by exon 1 (the normal protein has 625 amino acids). It is, therefore, unsurprising that sensitive immunoassay was unable to detect fibrinogen protein in the plasma of affected individuals in this family. Interestingly, a number of less severely truncated fibrinogen alpha chains have been described in hypofibrinogenemic patients, the smallest of which was due to a single base pair insertion in codon 268 (10).

To estimate the size of the deletions and to establish if they were, in fact, identical on all three mutant alleles, we performed Southern blotting analysis. Genomic DNA samples from all individuals from the affected family and from four normal controls were digested with BamHI and hybridized with a cloned probe pAFS1 generated by PCR amplification from the intergenic region between FGG and FGA (Fig. 4). The expected band of 13 $\mathrm{kb}$ was found for the control samples (Fig. 3, lanes 1-4), as well as for the obligate carriers (lanes 5-8). In addition, a larger band $(\sim 17 \mathrm{~kb})$ was obtained in these same carriers. Only the larger fragment was observed in the affected individuals (lanes 9-12). This abnormal fragment represents a deletion of $\sim 11 \mathrm{~kb}$ (which includes two BamHI sites and thus generates a band of paradoxically increased size). All three deletions were identical within the limits of the resolution of the technique.

Figure 4 depicts the genomic organization and $\mathrm{BamHI}$ restriction map of the fibrinogen gene cluster on chromosome 4 and summarizes the PCR and Southern data. The position of the telomeric end of the deletion is defined by the size of the deletion and by the BamHI restriction map (7). We conclude that the genetic defect in this family was a recurrent deletion of $\sim 11 \mathrm{~kb}$ of DNA that eliminates the majority of the FGA gene and thus leads to an absence of functional fibrinogen.

\section{Discussion}

The identification of the precise genetic defect of coagulation disorders is of value (a) to improve the differential diagnosis; (b) to permit early testing of other at-risk individuals; (c) to understand the correlation (if any) between genotype and clinical phenotype; $(d)$ to assist in therapeutic choices; and (e) to serve as an essential prerequisite for the development of new specific treatments, including gene therapy. Although congenital afibrinogenemia was originally described in 1920 (4) and the genetic defect assumed to be at the level of fibrinogen synthesis, no responsible gene and mutation had been identified. We found that the genetic defect in a nonconsanguineous Swiss family was a homozygous deletion of $\sim 11 \mathrm{~kb}$ eliminating the majority of the FGA gene. Importantly, the nature of the mutation we have identified finally confirms that humans can survive despite totally lacking the capacity to synthesize active fibrinogen and that these patients are genuinely afibrinogenemic, as opposed to being severely hypofibrinogenemic.

Typically, in rare autosomal recessive disorders, one expects to find patients who are either homozygous because of consanguinity or are compound heterozygotes. The four afibrinogenemia patients of the family presented here are homozygous for the FGA deletion but are nonconsanguineous; the mutant alleles have three $\boldsymbol{a}$
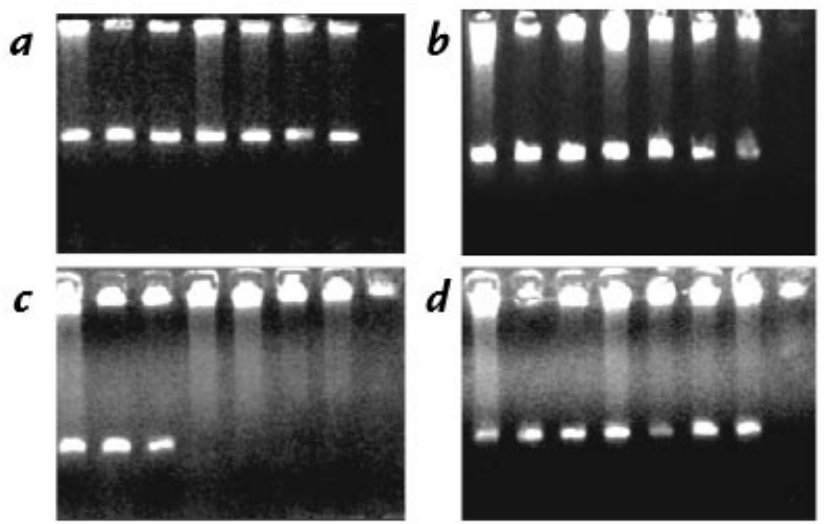

Figure 2

Representative PCR analyses of the extent of the fibrinogen locus deletion. DNA samples in lanes 1-3 were from obligate carriers (individuals 3-5 in Fig. 1) and in lanes 4-7 were from patients (individuals 6-9). Lane 8 contains the negative control (no DNA). The amplified fragments were: (a) FGG exon 7; (b) FGA exon 1; (c) FGA exon 5; and (d) FGB exon 7. $F G B$, fibrinogen beta-chain gene; FGG, fibrinogen gamma-chain gene.

different haplotypes according to closely linked flanking markers, suggesting that an identical mutation occurred independently three times in history. The existence of recurrent rearrangements has been described in a number of disorders and is commonly associated with inappropriate recombination between homologous sequences, for example, with the $F 8$ gene inversions in hemophilia A (11), CYP21B deletions in congenital adrenal hyperplasia (12), and alpha-globin gene deletions in alpha-thalassemias (13). Deletions can also be due to recombination between inverted Alu repeats, as in delta ${ }^{0}$ beta ${ }^{0}$-thalassemia (14), and familial hypercholesterolemia (15). Repetitive sequences that might be responsible for the recurrent deletion were sought in the FGA gene with the CENSOR program (16). Two such elements were identified in intron 1 of the FGA gene, the common MIR (mammalian-wide interspersed repeat; ref. 17) and an unusual $\mathrm{T}_{4} \mathrm{G}$ motif. PCR amplification of the FGA gene with a forward primer in exon 1 and a reverse primer in intron 1 situated between the two repeats revealed that the MIR was deleted in the affected individuals; therefore, this element is unlikely to be responsible for the recurrent mutation. The common mechanism of deletion in the fibrinogen locus will be definitively determined only by

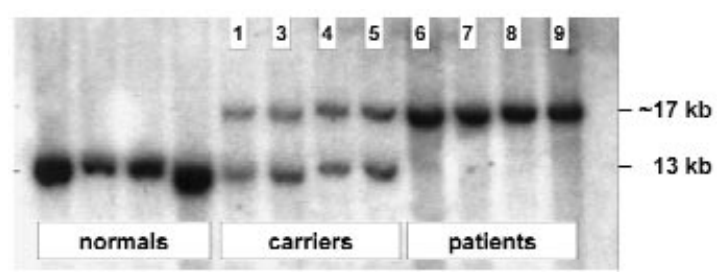

Figure 3

Southern blot analysis of the FGA deletion. The deletion appears as a band of paradoxically increased size because two BamHI sites are eliminated (see schematic in Fig. 4). Lanes 1-4, normal controls; lanes 5-8, obligate carriers; lanes 9-12, patients. Family members in lanes 5-12 are numbered according to the family tree in Fig. 1. 


\section{deletion}

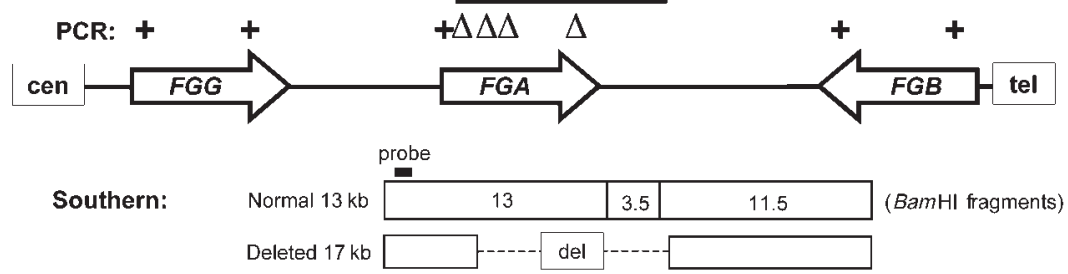

\section{Figure 4}

Schematic representation of the human fibrinogen gene cluster (after ref. 7). The arrows represent the gamma-, alpha-, and beta-chain genes and their $5^{\prime} \rightarrow 3^{\prime}$ orientation. The extent of the deletion is shown by the black bar. The results of the PCR analyses of the patients' DNA are summarized above the genes: + indicates fragment present; $\Delta$ indicates fragment absent (deleted). For details of the amplimers, see Table 1. The interpretation of the Southern analysis is shown below the genes. On normal chromosomes, the probe detects a $13-\mathrm{kb}$ Bam HI fragment, whereas the deletion removes two Bam $\mathrm{H} 1$ sites and thus leads to a paradoxically larger (17 kb) fragment.

sequencing of the breakpoints.

The fact that the congenital afibrinogenemia in this family is due to the deletion of a single small gene leads to obvious possibilities for gene therapy in the future, by delivery of the FGA cDNA or gene to the liver. The fact that the affected individuals have never made antibodies against their fibrinogen-replacement therapy is also encouraging. Furthermore, by a remarkable coincidence, there is already a knockout mouse model generated by a mutation similar to the human $F G A$ deletion initiating in intron 1 described here, i.e., the disruption of exon 1 of the FGA gene (18). These mice have no detectable liver FGA mRNA or circulating fibrinogen, although the levels of both $F G B$ and $F G G$ liver mRNAs are normal.

It will be interesting to determine the frequency of this mutation among other afibrinogenemia patients. We have examined three mutant alleles with different haplotypes, all of which have a common defect, and thus we predict that the FGA deletion will recur in many unrelated patients. However, it should be remembered that the first studies of the fibrinogen locus by Southern blotting did not detect deletions, implying that another mutation mechanism also exists, which may be allelic or due to further, hitherto unidentified, loci.

\section{Acknowledgments}

We thank all members of the family for their great enthusiasm, for donating blood samples, and for their continual practical help, without which this study would never have been possible. We are very grateful to Miha Furlan for the immunoassay data, to Phillippe de Moerloose, Bernhard Lämmle, and Kristina Peter for their invaluable assistance, to Anni Schönbörner for expert technical help, and to the many clinicians who aided in the collection of samples. We thank Hewlett-Packard (Geneva, Switzerland) for their kind donation of computer equipment. M. Neerman-Arbez is a recipient of a Marie Heim-Voegtlin grant from the Swiss National Science Foundation. This study was supported, in part, by the University and Cantonal Hospital of Geneva, and by the ZLB Central Laboratory.
1. Antonarakis, S.E., et al. 1995. Molecular etiology of factor VIII deficiency in hemophilia A. Adv. Exp. Med. Biol. 386:19-34.

2. Bertina, R.M., et al. 1994. Mutation in blood coagulation factor V associated with resistance to activated protein C. Nature. 369:64-67.

3. Nichols, W.C., et al. 1998. Mutations in the gene for ERGIC-53, a protein of the endoplasmic reticulum-Golgi intermediate compartment, cause combined deficiency of coagulation factors V and VIII. Cell. 93:61-68.

4. Rabe, F., and Salomon, E. 1920.Ueber-faserstoffmangel im Blute bei einem Falle von Hämophilie. Arch. Int. Med. 95:2-14.

5. Martinez, J. Congenital dysfibrinogenemia. 1997. Curr. Opin. Hematol. 4:357-365.

6. Crabtree, G.R. The molecular biology of fibrinogen. 1983. In The molecular basis of blood diseases. G. Stamatoyannopoulos, editor. W.B. Saunders. Philadelphia, PA. Chapter 17.

7. Kant, J.A., et al. 1985. Evolution and organization of the fibrinogen locus on chromosome 4: gene duplication accompanied by transposition and inversion. Proc. Natl. Acad. Sci. USA. 82:2344-2348.

8. Uzan, G., et al. 1984. Analysis of fibrinogen genes in patients with congenital afibrinogenemia. Biochem. Biophys. Res. Commun. 120:376-383.

9. Neerman-Arbez, M., et al. 1997. The locus for combined factor V-factor VIII deficiency (F5F8D) maps to 18q21, between D18S849 and D18S1103. Am. J. Hum. Genet. 61:143-150.

10. Ridgway, H.J., Brennan, S.O., Faed, J.M., and George, P.M. 1997. Fibrinogen otago: a major alpha chain truncation associated with severe hypofibrinogenaemia and recurrent miscarriage. Br. J. Haematol. 98:632-639.

11. Lakich, D., Kazazian, H.H., Jr., Antonarakis, S.E., and Gitschier, J. 1993. Inversions disrupting the factor VIII gene are a common cause of severe haemophilia A. Nat. Genet. 5:236-241.

12. Rumsby, G., Fielder, A.H., Hague, W.M., and Honour, J.W. 1988. Heterogeneity in the gene locus for steroid 21-hydroxylase deficiency. J. Med. Genet. 25:596-599.

13. Phillips, J.A., et al. 1980. Unequal crossing-over: a common basis of single alpha-globin genes in Asians and American blacks with hemoglobinH disease. Blood. 55:1066-1069.

14. Ottolenghi, S., and Giglioni, B. 1982. The deletion in a type of delta 0beta 0 -thalassaemia begins in an inverted AluI repeat. Nature. 300:770-771

15. Lehrman, M.A., et al. 1985. Mutation in LDL receptor: Alu-Alu recombination deletes exons encoding transmembrane and cytoplasmic domains. Science. 227:140-146.

16. Jurka, J., Klonowski, P., Dagman, V., and Pelton, P. 1996. CENSOR a program for identification and elimination of repetitive elements from DNA sequences. Comput. Chem. 20:119-121.

17. Smit, A.F., and Riggs, A.D. 1995. MIRs are classic, tRNA-derived SINEs that amplified before the mammalian radiation. Nucleic Acids Res. 23:98-102.

18. Suh, T.T., et al. 1995. Resolution of spontaneous bleeding events but failure of pregnancy in fibrinogen-deficient mice. Genes Dev. 9:2020-2033. 